\title{
Multiplexed Immunoassay: Quantitation and Profiling of Serum Biomarkers Using Magnetic Nanoprobes and MALDI-TOF MS
}

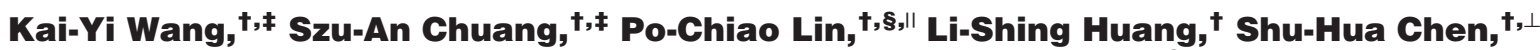 \\ Saib Ouarda, ${ }^{\dagger}$ Wen-Harn Pan, ${ }^{\otimes}$ Ping-Ying Lee, ${ }^{\circ}$ Chun-Cheng Lin, $, *, \mathcal{S}_{,},, \#$ and Yu-Ju Chen ${ }^{\star,+, \|, \perp, \#}$
}

\begin{abstract}
Institute of Chemistry, Institute of Biomedical Sciences, and Genomic Research Center and Chemical Biology and Molecular Biophysics, Taiwan International Graduate Program, Academia Sinica, Taipei, Department of Chemistry, National Central University, Taoyuan County, Department of Chemistry, National Tsing Hua University, Hsinchu, Department of Chemistry, National Taiwan University, Taipei, and Division of Cardiology, Department of Internal Medicine, Mackay Memorial Hospital, Mackay Medicine, Nursing and Management College, Taipei, Taiwan, ROC
\end{abstract}

Taking advantage of efficient affinity extraction by surfacefunctionalized magnetic nanoparticles (MNPs) and accurate MALDI-TOF MS readout, we present a multiplexed immunoassay for simultaneous enrichment and quantitation of multiple disease-associated antigens, serum amyloid A (SAA), C-reactive protein (CRP), and serum amyloid P (SAP) from human serum. To obtain reproducible MALDI signal response with direct on-MNP detection, the seed-layer method improved homogeneity of the cocrystallization of MNPs and captured antigens. Our methodology demonstrated good quantitation linearity of targeted analytes $\left(R^{2} \approx 0.97\right)$ with reduced signal variation (RSD $<10 \%$ ). The lower limit of quantitation is in the nanogram level with overall assay precision (intraday, $7.0 \%$; interday, $11.3 \%$ ) and accuracy (intraday, 6.3\%; interday, $\mathbf{1 7 . 5} \%$ ) including steps of nanoprobe extraction and MALDI-TOF MS analysis. This triplexed immunoassay showed overexpression of SAA and CRP in patients with cardiac catheterization or gastric cancer $(P<0.05)$, consistent with single-analyte ELISA and previous studies. Compared to the determination of disease onset by single protein quantitation, our multiplexed immunoassay revealed a distinct triplexed pattern in the control group, patients with gastric cancer, and cardiac catheterization. On the basis of the advantages of flexibility in nanoprobe preparation, high specificity and sensitivity, and rapid screening by MALDI-TOF MS, this platform may provide a new methodology for disease diagnosis.

* To whom correspondence should be addressed. Dr. Yu-Ju Chen, phone: +886-2-2789-8660. Fax: +886-2-2783-1237. E-mail: yjchen@chem.sinica.edu.tw. Dr. Chun-Cheng Lin, phone: +886-2-2789-8660. Fax: +886-2-2783-1237. E-mail: cclin66@nthu.edu.tw.

${ }^{\dagger}$ Institute of Chemistry, Academia Sinica.

* National Central University.

\& National Tsing Hua University.

" Chemical Biology and Molecular Biophysics, Taiwan International Graduate Program, Academia Sinica.

$\perp$ National Taiwan University.

${ }^{\otimes}$ Institute of Biomedical Sciences, Academia Sinica.

- Mackay Medicine, Nursing and Management College.

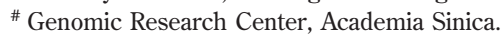

In the postgenome era, the field of proteomics promises to identify altered abundances or structures of tissue or fluid markers associated with human diseases, particularly cancer. The discovery and utility of biomarkers may provide earlier diagnosis and improved therapeutic intervention. Because of the complexity of human biology and the multifactorial nature of disease, it seems improbable that diseases could be predicted based on a single marker. Diagnoses using single-protein assays often result in insufficient specificity. Therefore, the development of multiplexed assays that can simultaneously screen multiple protein biomarkers is urgently needed for treatment response and drug discovery. ${ }^{1}$

To develop multiplexed assays, several multianalyte quantitation platforms have been reported for accurate clinical diagnosis, including the cytometric microbead assay, ${ }^{2}$ microarrays, ${ }^{3}$ and microfluidic devices. The cytometric bead assays consist of beads of different sizes, surface modifications, and fluorescent dyes to simultaneously measure and quantify multiple analytes in biological samples. ${ }^{2}$ Microarray assays based on the immobilization of multiple antibodies on solid supports followed by optical or radioactive detection ${ }^{4}$ have evolved in various formats such as glass slides, ${ }^{5}$ nitrocellulose films, ${ }^{6,7}$ agarose, ${ }^{8}$ or metal-coated slides. ${ }^{9}$ The antibody-based microarrays have been widely applied for the detection and quantitation of target antigens from biological fluids. ${ }^{10}$ Furthermore, microfluidic chips are sophisticatedly constructed platforms comprising microchannels for sample handling procedures, such as sample enrichment, incubation, and

(1) Kingsmore, S. F.; Patel, D. D. Curr. Opin. Biotechnol. 2003, 14, 74-81.

(2) Morgan, E.; Varro, R.; Sepulveda, H.; Ember, J. A.; Apgar, J.; Wilson, J.; Lowe, L.; Chen, R.; Shivraj, L.; Agadir, A.; Campos, R.; Ernst, D.; Gaur, A. Clin. Immunol. 2004, 110, 252-266.

(3) Kingsmore, S. F. Nat. Rev. Drug Discov. 2006, 5, 310-320.

(4) Schweitzer, B.; Kingsmore, S. F. Curr. Opin. Biotechnol. 2002, 13, 14-19.

(5) MacBeath, G.; Schreiber, S. L. Science 2000, 289, 1760-1763.

(6) Stillman, B. A.; Tonkinson, J. L. BioTechniques 2000, 29, 630-635.

(7) Kramer, A.; Feilner, T.; Possling, A.; Radchuk, V.; Weschke, W.; Burkle, L.; Kersten, B. Phytochemistry 2004, 65, 1777-1784.

(8) Afanassiev, V.; Hanemann, V.; Wolfl, S. Nucleic Acids Res. 2000, 28, E66.

(9) Zhu, H.; Bilgin, M.; Bangham, R.; Hall, D.; Casamayor, A.; Bertone, P.; Lan, N.; Jansen, R.; Bidlingmaier, S.; Houfek, T.; Mitchell, T.; Miller, P.; Dean, R. A.; Gerstein, M.; Snyder, M. Science 2001, 293, 2101-2105.

(10) Wilson, D. S.; Nock, S. Curr. Opin. Chem. Biol. 2001, 6, 81-85. 
separation. ${ }^{11}$ The reduction of sample consumption significantly improves the assay efficiency. For the above assays, optical methods including absorption, chemiluminescence, and fluorescence are the most widely adapted detection techniques, requiring additional chemical reactions or analyte-labeling steps. Moreover, highly specific antibodies are crucial for avoiding cross-reactivity between antibodies and nonantigenic proteins which lead to reduced specificity, sensitivity, and error-prone quantification. ${ }^{12}$ The most serious drawback is the inability to differentiate heterogeneous protein variants, which are not only quantitatively but also structurally dynamic. In addition to protein expression levels, the characterization of post-translational modifications may reveal correlation with disease progression. ${ }^{13,14}$

To achieve high specificity and sensitivity for heterogeneous protein structures and expression levels, recently, we have developed a new method, nanoprobe-based affinity mass spectrometry (NBAMS), for rapid antigen isolation and identification. ${ }^{15}$ The method provides good performance in biomarker analysis because the high surface area to volume ratio of nanoparticles increases the reaction activity, and the nature of magnetic nanoparticles (MNPs) in easy separations speeds up the assay procedure. Moreover, the high speed, high accuracy mass spectrometric analysis can reveal not only protein identification but also structural diversity. ${ }^{16,17}$ However, further application of NBAMS in clinical applications remains for further implementation in situations where protein quantitation is required to determine the disease onset. From a quantitative perspective, the major restriction is the poor reproducibility of signal intensity in MALDI MS analysis due to an inhomogeneous surface formed during sample/matrix crystallization which leads to "sweet spot" formation and increased signal fluctuation. To enhance the signal reproducibility in MALDI-TOF MS, fast evaporation of the matrix solution enhances homogeneity and facilitates quantitative analyses. ${ }^{18}$ Another strategy employs an internal standard to reduce the signal variation, instrument noise, or experimental procedures. ${ }^{19}$ Despite the success of these techniques to facilitate quantitative analysis in MALDI-TOF MS, these improvements as they relate to protein quantitation in complex biofluids require further evaluation.

In the present study, we explore the utility of NBAMS as a multiplexed immunoassay for multivariant biomarker profiling and quantitation. In contrast to conventional multiplexing through serial performance of single assays, our multiplexed assay is designed for multianalyte enrichment and identification using a mixture of antibody-conjugated MNPs (Ab@MNPs) against different antigens and direct MALDI-TOF MS readout without

(11) Dittrich, P. S.; Manz, A. Nat. Rev. Drug Discov. 2006, 5, 210-218.

(12) Phizicky, E.; Bastiaens, P. I.; Zhu, H.; Snyder, M.; Fields, S. Nature 2003, 422, 208-215.

(13) Biran, H.; Friedman, N.; Neumann, L.; Pras, M.; Shainkin-Kestenbaum, R. J. Clin. Pathol. 1986, 39, 794-797.

(14) Le, L.; Chi, K.; Tyldesley, S.; Flibotte, S.; Diamond, D. L.; Kuzyk, M. A.; Sadar, M. D. Clin. Chem. 2005, 51, 695-707.

(15) Chou, P. H.; Chen, S. H.; Liao, H. K.; Lin, P. C.; Her, G. R.; Lai, A. C.; Chen, J. H.; Lin, C. C.; Chen, Y. J. Anal. Chem. 2005, 77, 5990-5997.

(16) Lin, P. C.; Chou, P. H.; Chen, S. H.; Liao, H. K.; Wang, K. Y.; Chen, Y. J.; Lin, C. C. Small 2006, 2, 485-489.

(17) Chen, S. H.; Liao, H. K.; Chang, C. Y.; Juo, C. G.; Chen, J. H.; Chan, S. I.; Chen, Y. J. Proteomics 2007, 7, 3038-3050.

(18) Vorm, O.; Roepstorff, P.; Mann, M. Anal. Chem. 1994, 66, 3281-3287.

(19) van Kampen, J. J.; Burgers, P. C.; de Groot, R.; Luider, T. M. Anal. Chem. 2006, 78, 5403-5411. multiple steps of protein purification, elution, and amplification. We also introduce the seed-layer method ${ }^{20}$ and an exogenous internal standard to improve the homogeneity of sample crystallization in MALDI-TOF MS quantitation. To demonstrate the performance of this approach, serum amyloid A (SAA), C-reactive protein (CRP), and serum amyloid $\mathrm{P}$ component, which are present at different concentrations in human blood, were selected as model systems. CRP and SAA are both acute-phase response proteins that increase in concentration during inflammation and after tissue damage. SAA is a potential candidate for a gastric cancer-related marker, ${ }^{17,21,22}$ and CRP is well-known as a marker to predict cardiovascular disease. ${ }^{23,24}$ These proteins have received increased attention in disease diagnosis and biomedical application..$^{23,25,26}$ In addition to the evaluation of detection specificity, sensitivity and quantitation accuracy, the NBAMS immunoassay was implemented to quantitatively compare human serum samples from normal individuals and patients with cardiac catheterization or gastric cancer. We demonstrated that the multiplexed NBAMS immunoassay provides not only rapid and facile screening but also information on protein expression levels and post-translational modifications, all in a single assay.

\section{EXPERIMENTAL SECTION}

Materials. CRP, SAA, and SAP were purchased from Calbiochem (San Diego, CA). Cytochrome $c$, myoglobin, and sinapinic acid were purchased from Sigma-Aldrich (Mississauga, ON, Canada). Antihuman SAP polyclonal antibody, antihuman CRP monoclonal antibody, and antihuman SAA monoclonal antibody were purchased from DakoCytomation (Carpinteria, CA), Biodesign (Kennebunk, ME), and Abazyme (Needham, MA), respectively. The magnetic separator was purchased from Qiagen (Valencia, CA).

Human Serum Samples and ELISA Measurement. Serum samples from healthy subjects, patients with cardiovascular risk, patients with cardiac catheterization, and patients with gastric cancer were obtained with informed consent from the Department of General Surgery, Tri-Services General Hospital and Division of Cardiology, Department of Internal Medicine, Mackay Memorial Hospital in Taipei, Taiwan. A total of 29 healthy subjects, 15 patients with cardiovascular risk, 15 patients with cardiac catheterization, and 16 patients with gastric cancer were analyzed. Detailed patient information is presented in Supporting Information Table S-1. Serum samples were clotted for $30 \mathrm{~min}$ at room temperature and separated by centrifugation (Kubota, Osaka, Japan) at $1000 \mathrm{~g}$ for $10 \mathrm{~min}$ from blood samples, and the

(20) Onnerfjord, P.; Ekstrom, S.; Bergquist, J.; Nilsson, J.; Laurell, T.; MarkoVarga, G. Rapid Commun. Mass Spectrom. 1999, 13, 315-322.

(21) Juan, H. F.; Chen, J. H.; Hsu, W. T.; Huang, S. C.; Chen, S. T.; Yi-Chung Lin, J.; Chang, Y. W.; Chiang, C. Y.; Wen, L. L.; Chan, D. C.; Liu, Y. C.; Chen, Y. J. Proteomics 2004, 4, 2766-2775.

(22) Chan, D. C.; Chen, C. J.; Chu, H. C.; Chang, W. K.; Yu, J. C.; Chen, Y. J.; Wen, L. L.; Huang, S. C.; Ku, C. H.; Liu, Y. C.; Chen, J. H. Ann. Surg. Oncol. 2007, 14, 84-93.

(23) Pepys, M. B.; Hirschfield, G. M. J. Clin. Invest. 2003, 111, 1805-1812.

(24) Pearson, T. A.; Mensah, G. A.; Alexander, R. W.; Anderson, J. L.; Cannon, R. O., 3rd; Criqui, M.; Fadl, Y. Y.; Fortmann, S. P.; Hong, Y.; Myers, G. L.; Rifai, N.; Smith, S. C., Jr.; Taubert, K.; Tracy, R. P.; Vinicor, F. Circulation 2003, 107, 499-511.

(25) Kiernan, U. A.; Tubbs, K. A.; Nedelkov, D.; Niederkofler, E. E.; Nelson, R. W. FEBS Lett. 2003, 537, 166-170.

(26) Kiernan, U. A.; Nedelkov, D.; Tubbs, K. A.; Niederkofler, E. E.; Nelson, R. W. Proteomics 2004, 4, 1825-1829. 
supernatant was stored at $-80{ }^{\circ} \mathrm{C}$ until analyzed. The CRP concentration in serum was measured by the latex particleenhanced immunoephelometric assay using a nephelometer (Dade Behring, Marburg, Germany). The SAS 8.0 statistical software (SAS Institute, Heidelberg, Germany) was used for statistical analysis.

Preparation of Antibody-Conjugated Magnetic Nanoparticles. Antibodies were covalently conjugated to the MNP surface through the cross-linker bis( $N$-hydroxysuccinimide ester) (DSS). In brief, aminosilane-modified MNPs (1 mg) and DSS (10 mg) were dissolved in DMSO $(250 \mu \mathrm{L})$ solution for $2 \mathrm{~h}$ at room temperature. The resulting nanoparticles were washed with DMSO $(100 \mu \mathrm{L})$ three times to remove excess reagents. The nanoparticles resuspended with either antibody solution (anti-SAA, $4.4 \mathrm{mg} / \mathrm{mL}, 75 \mu \mathrm{L}$; anti-CRP, $15.4 \mathrm{mg} / \mathrm{mL}, 30 \mu \mathrm{L}$; anti-SAP, 6.7 $\mathrm{mg} / \mathrm{mL}, 50 \mu \mathrm{L})$. The mixture was incubated at $4{ }^{\circ} \mathrm{C}$ for $30 \mathrm{~min}$. Then, short methoxy-ethylene glycol was added to the mixture (for anti-SAA, $40 \mathrm{mM}, 75 \mu \mathrm{L}$; for anti-CRP, $60 \mathrm{mM}, 30 \mu \mathrm{L}$; for antiSAP, $80 \mathrm{mM}, 50 \mu \mathrm{L}$ ) and was followed by shaking for $12 \mathrm{~h}$ at 4 ${ }^{\circ} \mathrm{C}$. Finally, the $\mathrm{Ab} @ \mathrm{MNP}$ products were magnetically separated and washed extensively with PBS (0.1 M, pH 7.4) to remove excess antibodies. The final product was stored at $4{ }^{\circ} \mathrm{C}$ for further use.

Multiplexed Immunoaffinity Extraction. Three different antibody-conjugated MNPs (Ab@MNPs), anti-SAA, anti-CRP, and anti-SAP@MNPs, were prepared for the multiplexed immunoaffinity assay; the optimal quantity for the mixed Ab@MNPs was determined empirically as $5,3.75$, and $5 \mu \mathrm{g}$, respectively. Multiplexed immunoaffinity extraction was performed by incubation of $13.75 \mu \mathrm{g}$ of mixed $\mathrm{Ab} @ \mathrm{MNPs}$ with sample solution $(60 \mu \mathrm{L})$ at room temperature for $1 \mathrm{~h}$ with gentle rotation. After affinity extraction of target antigens, unbound nonantigenic molecules were removed by additional washing of the MNPs with $100 \mu \mathrm{L}$ of TTBS (Tween-TBS, 0.05\% Tween-20 in $150 \mathrm{mM} \mathrm{NaCl}$ and $20 \mathrm{mM}$ Tris-HCl, $\mathrm{pH}$ 7.4) and $100 \mu \mathrm{L}$ of deionized water, each twice. The antigen-captured MNPs were further treated by $10 \mathrm{ng}$ of myoglobin $(1 \mu \mathrm{L}, 10 \mathrm{mg} / \mathrm{L})$ as an internal standard and $2 \mu \mathrm{L}$ of matrix solution $(10 \mathrm{mg} / \mathrm{mL}$ sinapinic acid in 50:50:0.1 (v/v/v) acetonitrile/water/trifluoroacetic acid) for subsequent MALDI-TOF MS analysis.

For multiplexed protein quantitation in human serum, standard calibration curves were developed by a series of $60 \mu \mathrm{L}$ sample solutions containing SAA, CRP, and SAP. The calibrator solutions were protein mixtures composed of one calibrated protein (ranging from 2.5-100 ng) and two other target proteins at fixed concentrations (SAA, $30 \mathrm{ng}$; CRP, $3 \mathrm{ng}$; SAP, $300 \mathrm{ng}$ ) in a final volume of $60 \mu \mathrm{L}$ of PBS ( $\mathrm{pH}$ 7.4). For quantitative determination of these three proteins in serum from patients and normal individuals, $10 \mu \mathrm{L}$ of human serum was diluted with $50 \mu \mathrm{L}$ of PBS. The freshly prepared calibrators and serum samples were subjected to immunoaffinity extraction for subsequent MALDI-TOF MS analysis. We estimated that $12 \mu \mathrm{g}$ of SAP, $16 \mu \mathrm{g}$ of SAA, and $20 \mu \mathrm{g}$ of CRP can be retrieved by $1 \mathrm{mg}$ of anti-SAP@MNP, antiSAA@MNP, and anti-CRP@MNP, respectively. Furthermore, the evaluation of detection sensitivity in the human serum environment was studied by the preparation of the diluted serum sample (10 $\mu \mathrm{L}$ of serum in $50 \mu \mathrm{L}$ of PBS) with depletion of three target proteins using the Ab@MNPs. The precleaned serum sample was spiked with a known amount of standard proteins (2-20 ng) for analysis.

MALDI-TOF MS Analysis. Prior to pipetting the sample/ matrix mixture onto the MALDI sample plate, the seed-layer method was applied to create a predeposited matrix surface on a stainless steel well. ${ }^{20}$ In brief, diluted matrix solution $(1.5 \mathrm{mg} /$ $\mathrm{mL}$ sinapinic acid in 100:0.1 (v/v) acetone/trifluoroacetic acid) was freshly prepared, and $1 \mu \mathrm{L}$ was dropped onto the center of the MALDI sample well. Because of the fast evaporation of acetone, an even, dense, thin film of small matrix crystals was obtained in 1-2 s. The predeposited matrix homogeneity was examined by an optical microscope.

All MALDI-TOF MS spectra were obtained by Voyager DESTR (Applied Biosystems, Foster City, CA), equipped with a 337 $\mathrm{nm}$ nitrogen laser. The measurements were taken by a linear, delayed-extraction mode, using an accelerating voltage of $25 \mathrm{kV}$, $92 \%$ grid voltage, $0.3 \%$ guide wire voltage, 1000 ns delay time, and a low-mass gate of $5 \mathrm{kDa}$. A mixture of two standard proteins, cytochrome $c(12361 \mathrm{Da})$ and myoglobin (16 $952 \mathrm{Da})$, was used as external mass calibration references. The typical spectrum was obtained by averaging eight 50 -shot spectra followed by baseline correction and Gaussian smoothing using Data-Explorer software (Applied Biosystems).

\section{RESULTS AND DISCUSSION}

Experimental Design and Workflow. To demonstrate the feasibility of our NBAMS approach for multiplexed profiling of disease-associated proteins, three kinds of antibody-conjugated MNPs (designated as anti-SAA@MNPs, anti-CRP@MNPs, and anti-SAP@MNPs) were prepared for immunoaffinity capture of antigens in human serum. Figure 1 shows the implementation of NBAMS for multiplexed immunoassays. The mixture of three kinds of $\mathrm{Ab} @ \mathrm{MNPs}$ was incubated with human serum to facilitate antibody-antigen interaction. After removing the unbound, nonantigenic components from the surface of $\mathrm{Ab} @ \mathrm{MNPs}$ by a series of washing steps, the Ab@MNPs with captured antigens were then collected by magnetic separation, mixed with matrix, and spotted onto sample plates for direct MALDI-TOF MS analysis. The direct on-probe analysis of affinity bound analyte has gained increasing popularity in affinity MALDI MS analysis including microbead, ${ }^{27-31}$ affinity pipet tip, ${ }^{32,33}$ PVDF membrane, ${ }^{17}$ and nanoparticle. ${ }^{15,16}$ Without prior elution, analyte can be released from the affinity probe prior to matrix-analyte crystallization presumably due to dissociation from the acidity and organic nature of the matrix solution. $^{27,34}$ This strategy avoids the potential sample loss and interference from the elution salt that could reduce assay sensitivity and quantitative accuracy. Furthermore, the simple one-step

(27) Qian, X.; Zhou, W.; Khaledi, M. G.; Tomer, K. B. Anal. Biochem. 1999, $274,174-180$.

(28) Doucette, A.; Craft, D.; Li, L. Anal. Chem. 2000, 72, 3355-3362.

(29) Peter, J. F.; Tomer, K. B. Anal. Chem. 2001, 73, 4012-4019.

(30) Raska, C. S.; Parker, C. E.; Sunnarborg, S. W.; Pope, R. M.; Lee, D. C.; Glish, G. L.; Borchers, C. H. J. Am. Soc. Mass Spectrom. 2003, 14, 10761085.

(31) Warren, E. N.; Elms, P. J.; Parker, C. E.; Borchers, C. H. Anal. Chem. 2004, $76,4082-4092$.

(32) Niederkofler, E. E.; Tubbs, K. A.; Gruber, K.; Nedelkov, D.; Kiernan, U. A.; Williams, P.; Nelson, R. W. Anal. Chem. 2001, 73, 3294-3299.

(33) Kiernan, U. A.; Addobbati, R.; Nedelkov, D.; Nelson, R. W. J. Proteome Res. 2006, 5, 1682-1687.

(34) Papac, D. I.; Hoyes, J.; Tomer, K. B. Anal. Chem. 1994, 66, 2609-2613. 


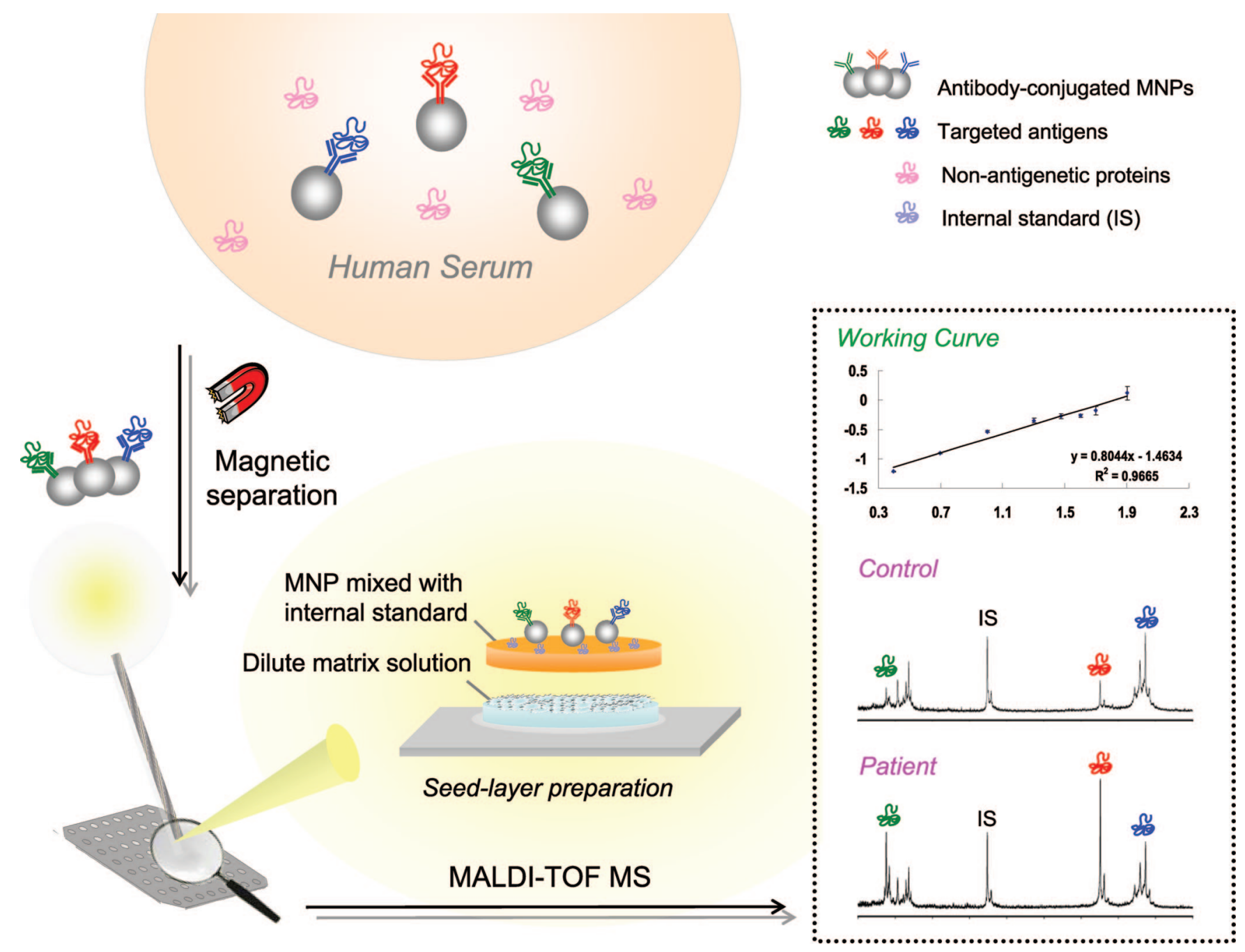

Figure 1. Schematic representation of selective enrichment and quantitative analysis of serum proteins by multiplexed NBAMS immunoassay.

purification/analysis greatly speeded up the NBAMS immunoassay; our multiplexed assay for rapid profiling of disease-associated proteins can be completed within $30 \mathrm{~min}$.

Multiplexed Profiling of Serum Proteins. To evaluate the specificity of our approach for detecting multiple targets, the NBAMS platform was applied to diluted human serum from a healthy individual. Prior to affinity extraction, a typical mass spectrum from a diluted human serum sample clearly displayed abundant proteins including human serum albumin, apolipoprotein A-I, hemoglobin $\alpha$-chain, hemoglobin $\beta$-chain, and transthyretin (Figure 2A). Signals from target antigens were absent due to their low abundance and the ion suppression effect. Immunoaffinity extraction using a mixture of anti-SAA@MNPs, anti-CRP@MNPs, and anti-SAP@MNPs produced strong signals of extracted SAA, $\mathrm{CRP}$, and SAP as shown in Figure 2B. None of the three $\mathrm{Ab} @ \mathrm{MNPs}$ nonspecifically captured other serum proteins. Our approach is clearly capable of analyzing multiple targets in a single assay without interference from abundant serum proteins.

Individual serum proteins are often heterogeneous, having various post-translationally modified and/or splice variant forms. ${ }^{35}$

(35) Nedelkov, D.; Kiernan, U. A.; Niederkofler, E. E.; Tubbs, K. A.; Nelson, R. W. Proc. Natl. Acad. Sci. U.S.A. 2005, 102, 10852-10857.
Mass spectrometric detection also revealed unique patterns of protein post-translational modifications that could not be differentiated by conventional immunoassay techniques. Compared to the single peaks of SAA and CRP at $m / z 11681$ and $23026 \mathrm{Da}$, the inserts in Figure 2B show multiple peaks originating from intact glycosylated SAP at $m / z 25460 \mathrm{Da}$, deglycosylated SAP missing one sialic acid residue (individual mass $=291 \mathrm{Da}$ ) at $\mathrm{m} / z 25171$ $\mathrm{Da}$, and deglycosylated SAP missing two sialic acid residues at $m / z 24882 \mathrm{Da}$ (also see Supporting Information Table S-2). These assignments were based on a mass measurement within $0.01 \%$ when compared with the calculated mass obtained from the protein sequence deposited in the Swiss-Prot data bank with potential modifications (theoretical intact SAP mass: $25462 \mathrm{Da}$ ). On the basis of the $<0.01 \%$ mass accuracy in routine assays, we found that the peak resolution and mass accuracy were not adversely affected by the presence of nanoparticles on the sample plate in the linear mode of MALDI-TOF MS analysis. The unique heterogeneously glycosylated structure of SAP has been unraveled and found to be important for the normal and pathophysiological function. ${ }^{17,26,36}$ Our result was consistent with previous literature, and glycosylation was further confirmed by specific recognition of sialic acid residue on SAP to wheat germ agglutinin (WGA) 


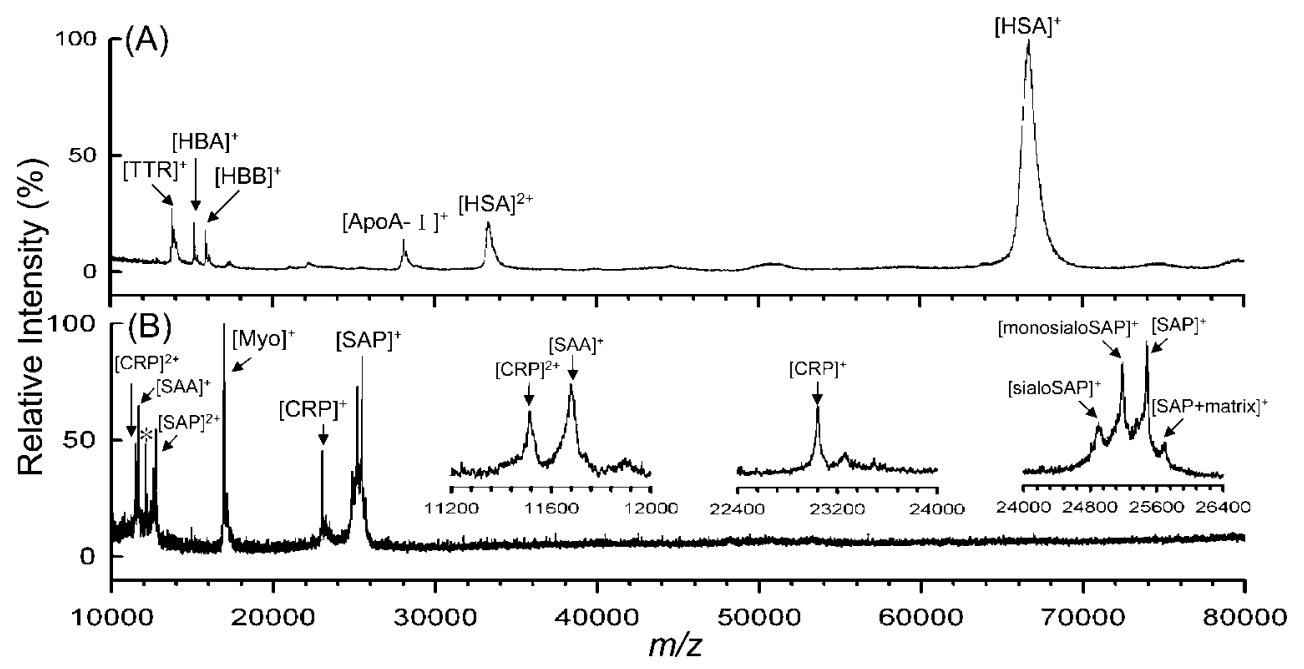

Figure 2. MALDI-TOF mass spectra obtained from (A) 300-fold-diluted human serum and (B) multiplexed NBAMS immunoassay for selective enrichment of SAA, CRP, and SAP in serum from a healthy individual. The insets of part B display detailed protein profiles for the target antigens. Myo, myoglobin, internal standard; HSA, human serum albumin; ApoA-I, apolipoprotein A-I; HBA, hemoglobin $\alpha$-chain; HBB, hemoglobin $\beta$-chain; TTR, transthyretin.

(data not shown). It is noted that an antibody specific to glycosylated SAP is not commercially available. Because of the difficulty to generate a modification-specific antibody for every protein, to date, it has not been possible to design an ELISA to simultaneously detect all post-translational modified forms. Compared with other immunoassays such as radioimmunoassay and ELISA, our approach highlights the advantage of mass spectrometry for detection of structurally distinct protein variants.

Evaluation of Detection Sensitivity. Detection sensitivity is crucial in the development of an immunoassay, especially in the detection of low-abundance antigens. Direct extraction of the target protein using the immobilized sample probe not only overcomes the suppression effect but also concentrates the target analytes onto the probe. To evaluate the detection limit of our approach for a human serum sample, serially diluted human serum, a $60 \mu \mathrm{L}$ sample solution containing $0.5-10 \mu \mathrm{L}$ of crude human serum from a healthy individual, was freshly prepared and incubated with functionalized MNPs. As shown in Figure 3, the singly and doubly charged SAP were the dominant peaks in all spectra, and the signals of SAA and CRP decreased progressively as the serum concentration decreased. These results demonstrate that the NBAMS immunoassay can concentrate multiple analytes from a highly diluted sample. On the basis of a signal-to-noise ratio of 3 for CRP, the detection limit of our multiplexed NBAMS assay was determined to be $0.5 \mu \mathrm{L}$ for human serum sample (Figure 3D).

To achieve a trustworthy estimation of detection limit with a known protein amount, we prepared target antigen-depleted serum samples and then spiked a known amount of standard protein. The results revealed that $2 \mathrm{ng}$ of SAP $(0.03 \mathrm{mg} / \mathrm{L})$ can be successfully retrieved from diluted human serum (see Supporting Information Figure S-1), which are comparable to the detection limit of the subnanogram level obtained in standard protein solution..$^{15}$ The results indicate high specificity of our approach that the assay performance is not interfered with by the presence

(36) Pepys, M. B.; Rademacher, T. W.; Amatayakul-Chantler, S.; Williams, P.; Noble, G. E.; Hutchinson, W. L.; Hawkins, P. N.; Nelson, S. R.; Gallimore, J. R.; Herbert, J.; et al. Proc. Natl. Acad Sci. U.S.A. 1994, 91, 5602-5606.

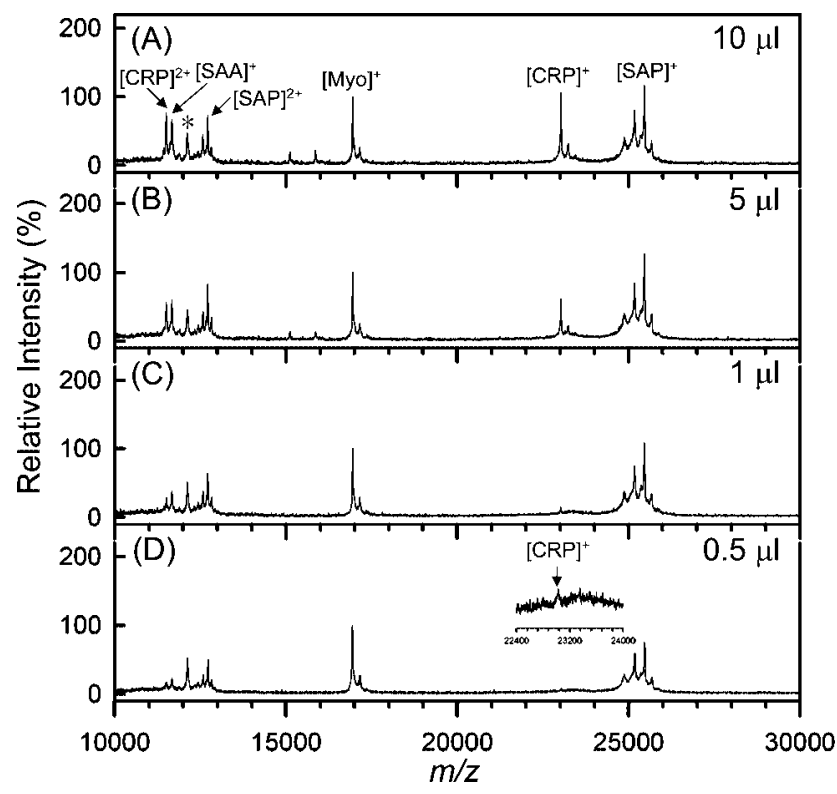

Figure 3. MALDI-TOF mass spectra of the three target antigens, SAA, CRP, and SAP, extracted from serially diluted serum samples (60 $\mu \mathrm{L}$ ) composed of (A) 10, (B) 5, (C) 1, and (D) $0.5 \mu \mathrm{L}$ of human serum from a healthy individual using the multiplexed NBAMS immunoassay. The internal standard, myoglobin, is designated as Myo. The $*$ represents the background signal generated from a commercial anti-SAP.

of a complex matrix. Considering that SAA, CRP, and SAP levels in serum from healthy subjects were $1-5,,^{37} 0.8-3,{ }^{23}$ and $30-40$ $\mathrm{mg} / \mathrm{L},{ }^{38}$ respectively, it is noteworthy the NBAMS immunoassay could detect SAA, CRP, and SAP in serum sample diluted $>100$ fold. Given the detection sensitivity of the nanoscale immunoassay at the nanomolar level, the antibody-conjugated MNPs offer the advantage of concentration effects to detect low-abundance and multiplexed target proteins from trace serum in samples.

(37) Pizzini, C.; Mussap, M.; Plebani, M.; Fanos, V. Scand. J. Infect. Dis. 2000, 32, 229-235.

(38) Pepys, M. B.; Dash, A. C.; Markham, R. E.; Thomas, H. C.; Williams, B. D.; Petrie, A. Clin. Exp. Immunol. 1978, 32, 119-124. 
(A)

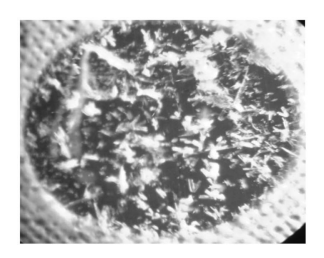

$(\mathrm{C})$

(B)

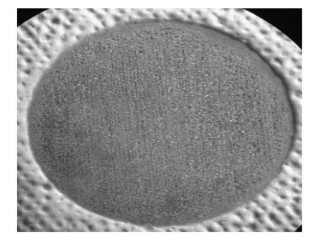

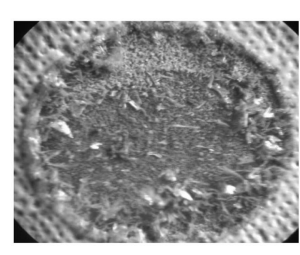

(D)

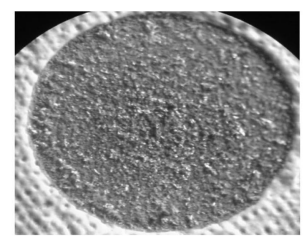

(E)

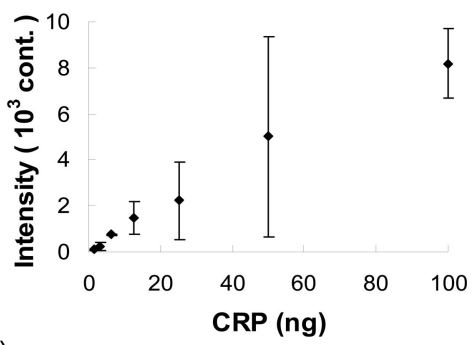

$(\mathrm{F})$

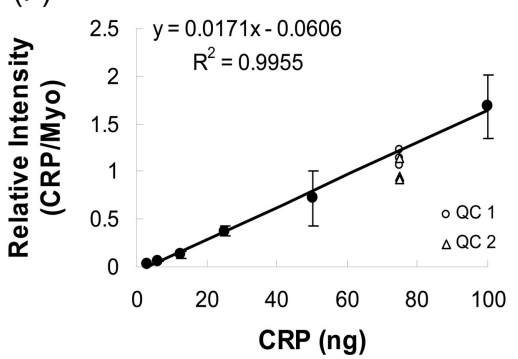

Figure 4. Improvement of the NBAMS assay using the seed-layer method and internal standard for homogeneity of matrix-sample crystallization and linear working curve. Parts A and B show the matrix crystal morphology (sinapinic acid) obtained from direct deposition of 50 ng of CRP before and after seed-layer preparation, respectively. The comparison of deposition of MNP-extracted CRP mixture before and after seed-layer preparation is shown in parts $C$ and $D$, respectively. The comparison of two standard calibration curves develop $(E)$ without and $(F)$ with the seed-layer method and an internal standard. The assay accuracy was evaluated by the quality control samples (QC 1, circle, intraday measurements; QC 2, triangle, interday measurements).

Improved Quantitation. Quantitative analysis relies on the stability of the analyte signal and reproducibility of the assay. Although MALDI-TOF MS is one of the most extensively used technologies for biomolecule identification, the poor reproducibility of signal intensities due to inhomogeneous cocrystallization of the analyte with matrix impedes its general applicability for quantitative studies. To improve signal reproducibility in MALDI MS analysis, a seed-layer method ${ }^{20}$ was applied to achieve a homogeneous sample/crystal surface. The effect of the seed-layer method is shown in Figure $4 \mathrm{~A}-\mathrm{D}$. The crystal morphologies prepared by the conventional dried-droplet method resulted in an inhomogeneous distribution of sample/matrix crystals for both direct protein deposition (50 ng CRP, Figure 4A) and MNPextracted analyte (50 ng CRP, Figure $4 \mathrm{C}$ ). In contrast, the seedlayer surface significantly improved the sample homogeneity in both the conventional MALDI sample plate (Figure 4B) and the MNP-depositing surface (Figure 4D). The formation of a uniform, homogeneous crystal can be attributed to the better crystallization of the matrix and complex of antigens and Ab@MNPs on the seed-layer surface. In an attempt to achieve maximum signal reproducibility, we also optimized the spectrum accumulation method to minimize signal variation. Stable signals with less than $10 \%$ RSD on MALDI-TOF MS analysis could be obtained by accumulating 400 shots from eight MALDI target positions.

Encouraged by the improved sample homogeneity and concomitant signal reproducibility, we further pursued the feasibility of multiplexed quantitation by NBAMS. Serial dilution of CRP protein was prepared and processed with NBAMS to plot a working curve for quantitation. Figure 4 shows the comparison of quantitative working curves developed with or without an internal standard and seed-layer preparation. As shown in Figure $4 \mathrm{E}$, the curve calculated based on peak height showed neither good signal linearity nor signal stability. We also evaluated the
Table 1. Summary of Intra- And Interday Precision and Accuracy in NBAMS Immunoassay

\begin{tabular}{cccccc} 
intraday & $\begin{array}{c}\text { nominal } \\
\text { amount (ng) }\end{array}$ & $\begin{array}{c}\text { observed } \\
\text { amount (ng) }\end{array}$ & $\begin{array}{c}\text { average } \\
\text { (ng) }\end{array}$ & $\begin{array}{c}\text { precision } \\
(\%)\end{array}$ & $\begin{array}{c}\text { accuracy } \\
(\%)\end{array}$ \\
& 75.0 & 75.4 & $70.3 \pm 9.0$ & 7.0 & 6.3 \\
interday & 75.0 & 69.8 & & & \\
& & 65.6 & & & \\
& & 69.9 & $61.9 \pm 12.9$ & 11.3 & 17.5 \\
& 58.6 & & & \\
\hline
\end{tabular}

relative quantitative method using the signal intensity ratio of the analyte signal to an internal reference standard. As shown in Figure $4 \mathrm{~F}$, the working curve calculated based on peak height ratio of analyte to internal standard had a good linear response over at least 1 order of magnitude $(3.125-100 \mathrm{ng})$. Significantly reduced signal fluctuation $(\mathrm{RSD}<10 \%)$ and good linearity $\left(R^{2}=\right.$ $0.995)$ could be achieved with a seed-layer surface and the addition of an internal standard. Accordingly, the superior reproducibility provides convincing evidence that quantitative analysis by NBAMS can incorporate the improved seed-layer modified surface, which facilitates automatic data acquisition and reliable quantitation linearity.

A reliable quantitative assay relies on high reproducibility and high accuracy. To evaluate the assay precision and accuracy of our approach, the intra- and interbatch precision and accuracy were studied by a set of quality control samples with $75 \mathrm{ng}$ of CRP (three triplicate measurements for each experiment, shown in Figure $4 \mathrm{~F}$ ). As detailed in Table 1, a 7.0\% precision and 6.3\% accuracy were obtained within a single day assay and an $11.3 \%$ precision and $17.5 \%$ accuracy were obtained for interday assays. On the basis of the validation standard from the Food and Drug Administration (FDA), a valid bioanalytical method has to fulfill the criteria that percent deviation from the nominal concentration (accuracy) and the relative standard deviation (precision) have 


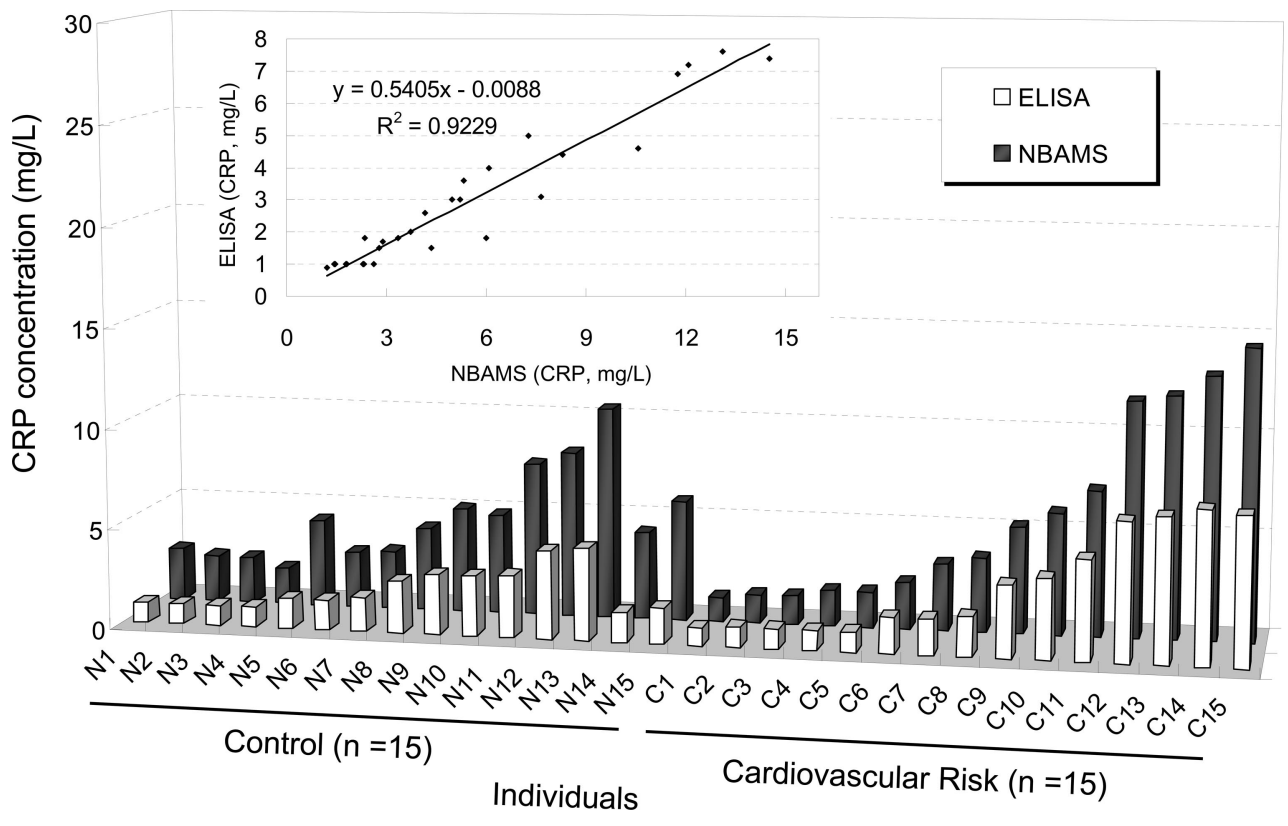

Figure 5. The CRP concentrations determined by NBAMS were compared with those obtained from ELISA for 15 control and 15 cardiovascular risk individuals. The inset shows the linear correlation between these two methods.

to be better than $20 \%{ }^{39}$ Therefore, the data demonstrated good reproducibility and accuracy of our approach as an immunoassay.

Comparison of the NBAMS Immunoassay with ELISA. We further implemented NBAMS as a quantitative immunoaffinity assay for selected proteins in serum samples between healthy subjects and patients. The comparison was performed for two groups of human serum samples from controls $(N=15)$ and from individuals with cardiovascular risk who had at least two risk factors among hypertension, stroke, smoking, or diabetes $(N=$ 15). By extrapolating the data from the calibration curves shown in Figure $4 \mathrm{~F}$, the $\mathrm{CRP}$ concentrations in normal individuals and cardiovascular risk individuals could be determined (Figure 5). To evaluate the reliability of the quantitative analysis in our approach, CRP was analyzed in parallel by ELISA. The CRP concentrations measured by NBAMS correlated with that measured by ELISA. Both measurements showed the same trend in individual-to-individual variation; similar CRP concentrations were obtained for the normal controls (NBAMS, $1.2-14.5 \mathrm{mg} / \mathrm{L}$; ELISA, $0.9-7.4 \mathrm{mg} / \mathrm{L}$ ) and cardiovascular risk patients (NBAMS, 1.8-10.6 $\mathrm{mg} / \mathrm{L}$; ELISA, $1.0-4.6 \mathrm{mg} / \mathrm{L}$ ). Interestingly, the mean CRP concentrations of individuals with cardiovascular risk (NBAMS, $4.7 \pm 2.5 \mathrm{mg} / \mathrm{L}$; ELISA, $2.2 \pm 1.2 \mathrm{mg} / \mathrm{L}$ ) were slightly lower than the normal individuals (NBAMS, $5.8 \pm 4.8 \mathrm{mg} / \mathrm{L}$; ELISA, $3.5 \pm$ $2.7 \mathrm{mg} / \mathrm{L}$ ). We note that several control individuals have high CRP levels, and therefore no significant difference in average CRP concentration was observed between the control group and cardiovascular risk group. The inset of Figure 5 shows the correlation of these two methods; the good linearity indicates the same trend in protein expression measurements. The discrepancies between NBAMS and ELISA may be attributable to limitations on the antibody-antigen interaction in the solid surface of ELISA. NBAMS takes advantage of a more effective immunoaffinity extraction in solution. In addition, any false positive signal arising from nonantigenic attachment can be differentiated based on a

(39) Guidance for industry: Q2B Validation of Analytical Procedure, Methodology, http//www.fda.gov/cber/guidelines.htm, 1996. characteristic mass-to-charge ratio. The comparison highlights the advantage of a mass spectrometry-based immunoassay with regard to specific signal response and accurate identification, which are critical for quantitative analysis.

Multiplexed Protein Quantitation for Differential Serum Protein Expression in Patients and Normal Individuals. The screening of specific biomarkers is important for early cancer diagnosis. Single-marker analyses typically lack sensitivity and specificity, however, thereby compromising their diagnostic accuracy. Accordingly, combining multiple markers is logical for a more valid prediction of the biological outcome, improving the diagnostic performance..$^{40}$ Moreover, multiplexed immunoassay presents advantages of reduced analysis time, lower cost, and smaller sample size. ${ }^{3}$

Encouraged by the high correlation of NBAMS with ELISA, we further evaluated the feasibility of multiplexed pattern recognition and protein quantitation in a clinical setting. A proof-of-concept experiment was performed by quantitative comparison of 60 serum samples, including 29 healthy controls, 15 patients with cardiac catheterization, and 16 patients with gastric cancer. Gastric cancer is one of the most common malignancies and is a leading cause of cancer-related deaths worldwide. ${ }^{41}$ Although the incidence and mortality of gastric cancer have declined over the past 40 years and advances in early diagnosis provide excellent long-term survival probabilities for early stage patients, the overall 5-year survival rate still remains $<10 \% .{ }^{42}$ The overexpression of acutephase protein, SAA $(<5 \mathrm{mg} / \mathrm{L}$ in healthy individuals), was observed in sera of gastric cancer patients and was identified as a potential serum biomarker. ${ }^{17,21,22}$ Among 1.5 million heart attacks in the United States each year, half of apparently healthy individuals have normal cholesterol levels. ${ }^{43}$ For better prediction of risk of heart disease, CRP production, another acute-phase

(40) Etzioni, R.; Urban, N.; Ramsey, S.; McIntosh, M.; Schwartz, S.; Reid, B.; Radich, J.; Anderson, G.; Hartwell, L. Nat. Rev. Cancer 2003, 3, 243-252. (41) Parkin, D. M.; Pisani, P.; Ferlay, J. Int. J. Cancer 1999, 80, 827-841.

(42) Peddanna, N.; Holt, S.; Verma, R. S. Anticancer Res. 1995, 15, 2055-2064.

(43) Ridker, P. M. Circulation 2003, 108, e81-85. 


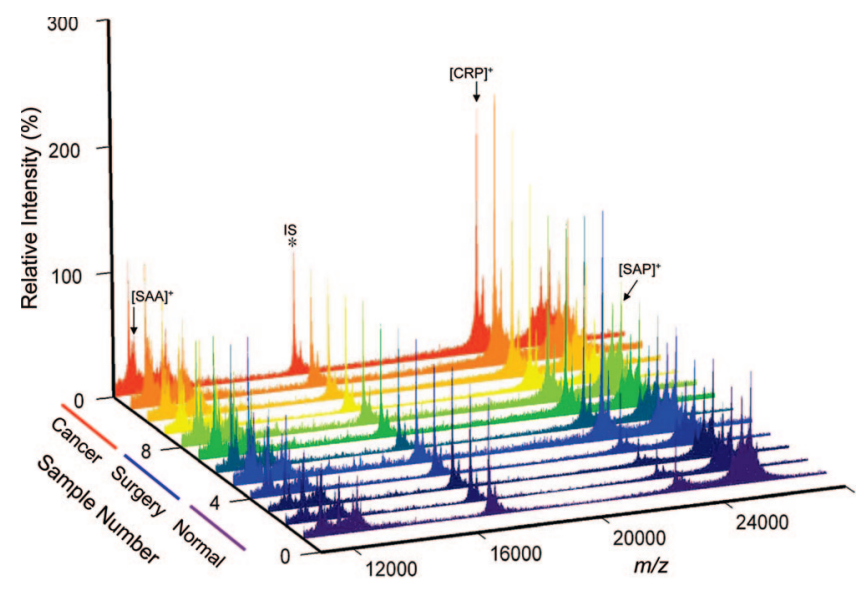

Figure 6. The mass spectral profile of SAA, CRP, and SAP from healthy individuals (Normal), patients with cardiac catheterization (Surgery), and patients with gastric cancer (Cancer). The $*$ represents the peak from the internal standard (IS), myoglobin.

response, has been hypothesized as a marker to predict the potential risk of cardiovascular disease..$^{23,24}$

Figure 6 shows representative spectra of 12 samples, including 4 normal controls, 4 patients with cardiac catheterization (designated as Surgery), and 4 patients with gastric cancer (designated as Cancer), obtained by the multiplexed NBAMS immunoassay. The three target antigens, SAA, CRP, and SAP, were detected in all subjects, despite the fact that the CRP levels in a few of the healthy individuals were below the detection limit of ELISA $(<0.159 \mathrm{mg} / \mathrm{L}) .{ }^{21}$ As shown in Figure 6, the relative levels of CRP and SAA were considerably higher in patients with cardiac catheterization and gastric cancer than in the controls, whereas the concentrations of SAP were relatively constant across all subjects. ${ }^{38}$ The distinct profiles between the three groups are consistent with the prognostic significance reported previously..$^{22,23}$

We also measured the concentrations of the three target proteins in human serum. A series of standard protein solutions composed of varying amounts of SAA, CRP, and SAP were prepared and processed by the multiplexed NBAMS immunoassay to establish standard calibration curves. The working curves using relative peak intensity displayed in Figure 7 show a good linear relationship with protein content $\left(R^{2}=0.978\right.$ for SAA, $R^{2}=0.993$ for $\mathrm{CRP}$, and $R^{2}=0.967$ for $\mathrm{SAP}$ ). The lower limits of quantitation (LLOQ) are 5, 5, and $2.5 \mathrm{ng}$ and the upper limits of quantitation (ULOQ) are 100, 80, and $80 \mathrm{ng}$ for SAA, CRP, and SAP, respectively, over at least 1 order of magnitude. The limit of detection (LOD) of target protein can be readily achieved at the nanogram level. To evaluate the quality of the linear regression lines, we calculated the standard error of estimate (SEE) and relative standard error of estimate (RSEE). The SEE and RSEE for the calibration curves are 0.057 and $9.7 \%$ for SAA; 0.095 and $24.8 \%$ for CRP; and 0.101 and $38.8 \%$ for SAP. The results demonstrated the adequate linearity ranges and assay sensitivities of our approach in simultaneous quantitation of these diseaseassociated proteins in human serum.

By extrapolating the relative intensities from the working curves, we calculated the concentrations of SAA, CRP, and SAP in normal individuals and patients with gastric cancer and cardiac catheterization (see the detailed information of all subjects in
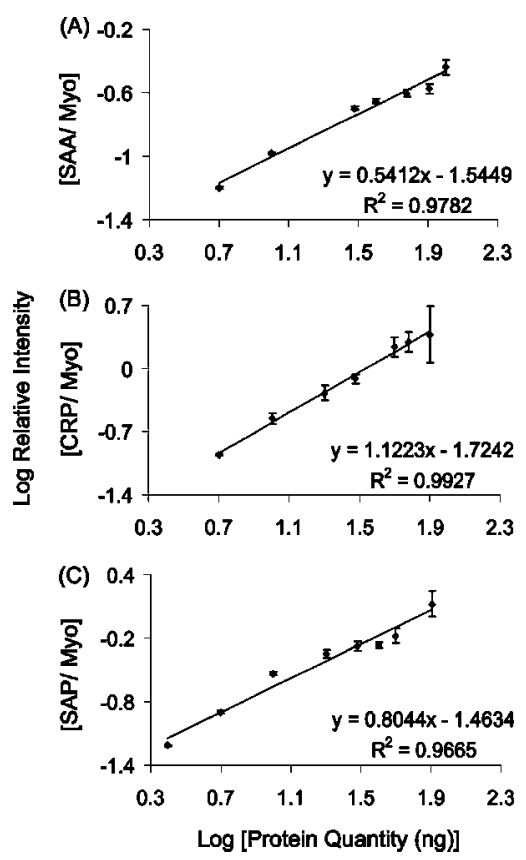

Figure 7. Standard calibration curves for (A) SAA, (B) CRP, and (C) SAP constructed using relative ion intensity normalized to the ion intensity of myoglobin.

Supporting Information Table S-3). Figure 8A shows scatter plots of individual protein concentrations for different groups. The levels of CRP and SAA were significantly different between control and patient groups $(P<0.05 ; t$ test $)$. The results showed the differential levels of CRP in healthy controls $(2.33 \pm 0.31 \mathrm{mg} / \mathrm{L})$ and in patients with cardiac catheterization $(6.43 \pm 0.46 \mathrm{mg} / \mathrm{L})$, which is consistent with the cardiovascular risk prediction of $\geq 3$ $\mathrm{mg} / \mathrm{L}$ for high-risk groups published by the Centers for Disease Control and Prevention and the American Heart Association. ${ }^{24}$ Similarly, the higher levels of CRP observed in gastric cancer patients $(7.28 \pm 1.04 \mathrm{mg} / \mathrm{L})$ is in agreement with previous studies. ${ }^{44}$ The SAA level in controls $(4.14 \pm 0.54 \mathrm{mg} / \mathrm{L})$ corresponds to the basal concentration of $1-5 \mathrm{mg} / \mathrm{L}$ in human serum, ${ }^{37}$ whereas the SAA level was significantly higher in patients with cardiac catheterization (up to $8.89 \pm 1.31 \mathrm{mg} / \mathrm{L}$ ) and gastric cancer (up to $9.77 \pm 1.47 \mathrm{mg} / \mathrm{L}$ ). It has been reported that SAA concentration can reflect disease states and tumor progression in gastric, lung, and ovarian cancer. ${ }^{13,21,22,45,46}$ In our recent study on gastric cancer, significant overexpression of SAA correlated better with diagnostic accuracy compared with conventional tumor markers, suggesting that SAA is not only a potential marker for malignancy but also a valuable indicator for postoperative followup. ${ }^{22}$ Even with the relatively small number of patients, our results are consistent with the previous studies, clearly demonstrating the feasibility of our multiplexed NBAMS approach in multiple biomarker screening, allowing for direct comparison of expression levels of different disease-associated serum proteins across various disease states.

(44) Solakidi, S.; Dessypris, A.; Stathopoulos, G. P.; Androulakis, G.; Sekeris, C. E. Clin. Biochem. 2004, 37, 56-60.

(45) Howard, B. A.; Wang, M. Z.; Campa, M. J.; Corro, C.; Fitzgerald, M. C., Jr Proteomics 2003, 3, 1720-1724.

(46) Moshkovskii, S. A.; Serebryakova, M. V.; Kuteykin-Teplyakov, K. B.; Tikhonova, O. V.; Goufman, E. I.; Zgoda, V. G.; Taranets, I. N.; Makarov, O. V.; Archakov, A. I. Proteomics 2005, 5, 3790-3797. 
(A)

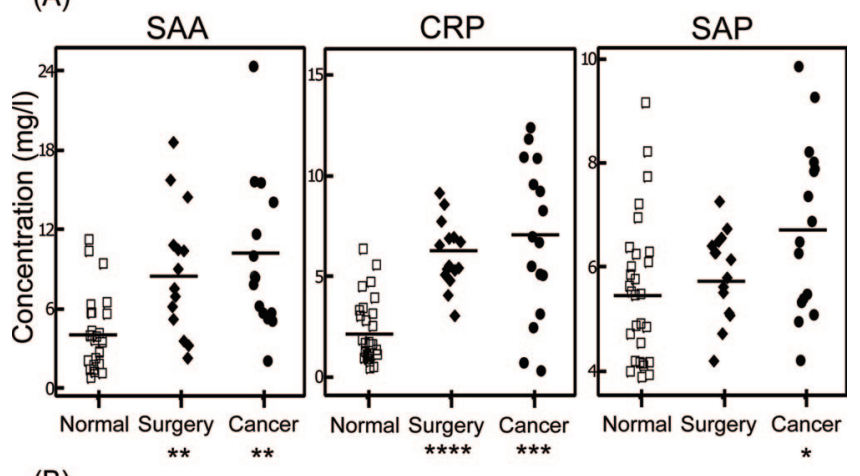

(B)

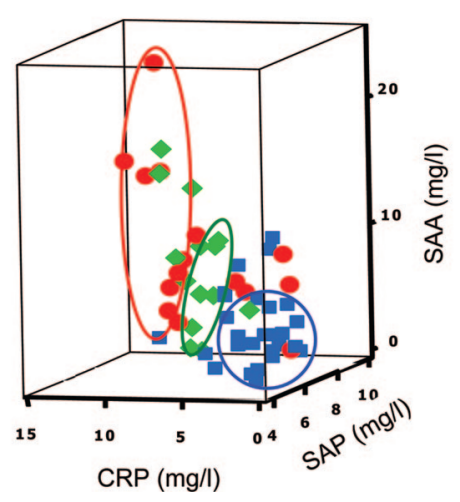

Figure 8. (A) Comparison of SAA, CRP, and SAP concentrations in healthy individuals ( $\square$, Normal), patients with cardiac catheterization $(\diamond$, Surgery), and patients with gastric cancer ( $\bullet$, Cancer). The horizontal bar represents the mean concentration. Asterisks denote statistically significant differences between control and patient groups: $*, P<0.01 ; * *, P<0.005 ; * * *, P<0.001 ; * * * *, P<0.0001$. (B) Three-coordinate scatterplot for target protein concentrations measured by the multiplexed NBAMS immunoassay for three groups: control (blue squares), Surgery (green diamonds), and Cancer (red circles).

The disease-specific differences in expression levels are very subtle and may require a panel of markers for diagnosis. Accordingly, the overlap of multiple biomarkers complements assays based on a single marker. Figure $8 \mathrm{~B}$ shows a threecoordinate scatterplot of the protein concentration obtained by multiplexed NBAMS immunoassay. The triplexed protein pattern showed that control subjects clustered at lower concentrations, whereas the patient groups were distributed at higher values. The "virtual diagnosis pattern" revealed by the triplexed screening of three sample groups demonstrates that the multiplexed immunoassay yields a more valid pattern in the determination of the physiological status. We expect that a larger number of clinical samples may provide a more obvious multivariate analysis pattern to improve disease diagnosis. Most importantly, the choice of protein marker plays a critical role in validity of multivariate analysis for specific disease diagnosis, an aspect that we are currently investigating.

\section{CONCLUSIONS}

In this study, NBAMS was implemented as a multiplexed immunoassay for quantitative analysis to determine the disease onset. The simple and rapid assay provides excellent target antigen enrichment as well as multiplex quantitation. The differential expression level of SAA, CRP, and SAP could be simultaneously quantified within $30 \mathrm{~min}$. Better than $20 \%$ precision and accuracy demonstrated high reproducibility and accuracy of our approach as a reliable immunoassay. By incorporation of a panel of markers into the triplexed assay, the multivariate differentiation emphasizes the use of multiple markers to increase diagnostic accuracy. With rapid screening by MALDI-TOF MS, our multiplexed immunoassay represents a new platform with the potential for integration into high-throughput protein assays and clinical diagnoses. Given the rapidly increasing use of protein assays with diverse applications, our NBAMS assay offers a flexible design for immobilization of molecular probes on a nanoparticle surface. Beyond antigen extraction by antibodies, extended applications of affinity capture via protein, small molecules, or other biomolecules can be performed on surface-functionalized nanoparticles. Given the versatile assay design, stable affinity nanoprobe, simple analytical procedure, and compatibility with direct MALDI-TOF MS analysis, our newly developed methodology may be useful for rapid target protein quantitation, characterization of protein modification, and other applications in clinical proteomics.

\section{ACKNOWLEDGMENT}

This work was supported by the Technology Program for Nanoscience and Nanotechnology of Academia Sinica and the National Science Council, Taiwan. We greatly appreciate the use of the MALDI-TOF MS at the Institute of Biomedical Sciences, Academia Sinica, Taiwan, and samples of patients with gastric cancer from Dr. Jenn-Han Chen at Tri-Services General Hospital, Taiwan.

\section{SUPPORTING INFORMATION AVAILABLE}

Evaluation of detection sensitivity in human serum, information for patients and healthy controls, molecular mass, and average concentration of three target serum proteins identified by the multiplexed NBAMS immunoassay. This material is available free of charge via the Internet at http://pubs.acs.org.

Received for review February 20, 2008. Accepted June 9, 2008.

AC800354U 